\title{
Réaliser une recherche socialement juste en éducation : apports et limites d'une approche épistémo- méthodologique féministe
}

\author{
Julie Larochelle-Audet, Université de Montréal \\ Marie-Odile Magnan, Université de Montréal
}

DOI : 10.51186/journals/ed.2021.11-1.e467

\section{Résumé}

Cet article présente l'approche épistémo-méthodologique féministe constituée pour élaborer et mener une recherche qualitative sur l'organisation et la reproduction des rapports de domination dans les distributions dissymétriques du travail enseignant au Québec (Larochelle-Audet, 2019). II expose les principaux postulats épistémologiques, outils méthodologiques et courants théoriques mobilisés afin de répondre aux exigences d'une recherche socialement juste (Strega \& Brown, 2015a). La recherche a été réalisée à l'aide de l'ethnographie institutionnelle (Smith, 2018), depuis le point de vue d'enseignantes de groupes racisés en début de carrière (14). Leurs récits, recueillis lors d'entretiens semidirigés, ont guidé l'exploration de processus et pratiques régulant leur sélection et leur évaluation en emploi, à partir d'entretiens menés avec des directions d'école et d'autres employées clés du réseau scolaire public et d'une analyse documentaire du cadre législatif. Comme cette méthode d'enquête sociologique tend à surexposer les activités des groupes dominants et leurs institutions, la pensée féministe noire a été mobilisée pour légitimer les expériences des enseignantes de groupes racisés en tant que savoirs. Les extraits de leurs récits présentés montrent comment se co-forment les systèmes d'oppression dans l'organisation du travail enseignant et différentes stratégies mises en œuvre pour y résister.

Mots-clés : division du travail enseignant, ethnographie institutionnelle, idéologie raciste, justice sociale, pensée féministe noire

\section{Abstract}

This article presents the feminist framework used to develop and conduct qualitative research on the organization and reproduction of power relationships in the asymmetrical distributions of teaching work in Québec (Larochelle-Audet, 2019). It sets out the main epistemological assumptions, methodological tools and theoretical currents mobilized to meet the requirements of socially just research (Strega \& Brown, 2015a). The research, informed by institutional ethnography (Smith, 2018), builds on the points of view of teachers from 
racialized groups at the start of their careers (14). Their accounts, collected in semi-directed interviews, guided the exploration of the processes and practices that regulated their selection and the evaluations of their work, based on interviews with school principals and other key employees in the public school network and on a documentary analysis of the legislative framework. As this method of sociological inquiry tends to overexpose the activities of dominant groups and their institutions, we mobilized Black feminist thought to legitimize the experiences of teachers from racialized groups as knowledge. Excerpts from their accounts show how oppressive systems are co-formed in the structure of teaching work, and the different strategies used to resist them.

Keywords: black feminist thought, division of teaching work, institutional ethnography, racist ideology, social justice

\section{INTRODUCTION}

Au cours des dix dernières années, un corpus croissant de recherches s'est intéressé à l'insertion professionnelle du personnel enseignant immigrant au Canada, et notamment au Québec (Duchesne, 2017 ; Morrissette \& Demazière, 2018 ; Niyubahwe, 2015). Les travaux menés en français sur cette thématique partagent généralement un même point de départ : les difficultés rencontrées par le personnel enseignant immigrant - en particulier les personnes ayant été qualifiées à l'extérieur du Canada ou ayant immigré depuis moins de dix ans - au moment d'intégrer le milieu scolaire. Une analyse critique de ce corpus a révélé la présence de cadres d'interprétation essentialisant le personnel enseignant immigrant et leurs compétences professionnelles (Larochelle-Audet, 2017 ; 2019). Ce qui est jugé problématique dans leur travail est fréquemment expliqué à partir de déficits " culturalistes », qui mettent "l'accent sur une définition figée de la culture de l'immigrant en tentant de le catégoriser selon des caractéristiques "objectives" liées à ses supposés comportements, modes de vie, pratiques et coutumes » (Rachédi \& Vatz Laaroussi, 2021, p. 91).

La mise au jour de cadres d'interprétation axés sur les déficits dans ce corpus de recherches spécifiques nous a conduites à explorer de nouvelles orientations épistémologiques, théoriques et méthodologiques pour initier une recherche ${ }^{1}$ sur l'expérience d'insertion professionnelle de ces enseignantes ${ }^{2}$. Nous avons explicitement endossé un positionnement épistémologique interprétatif-critique engagé pour la justice sociale, c'est-à-dire orienté vers

\footnotetext{
1 Nous souhaitons remercier les évaluateurs/trices de l'article pour leurs commentaires, qui ont permis de bonifier à plusieurs égards notre article, et mentionner que cette recherche a été financée par le Conseil de recherches en sciences humaines (CRSH) et le Fonds de recherche du Québec - Société et culture (FRQSC).

2 Dans ce texte, le terme "enseignantes " englobe à la fois les femmes et les hommes exerçant cette profession. Ce choix traduit la prédominance des femmes en enseignement au Québec $-75 \%$ en 2019 (Ministère de l'Éducation, 2019, cité par Gagnon, 2019, 25 septembre) - et un positionnement féministe visant à visibiliser les femmes.
} 
la production de connaissances utiles à l'émancipation des individus et à la transformation des institutions où se reproduit la domination (Potts \& Brown, 2015). Selon Hamrouni (2012), la domination est " le phénomène par lequel des contraintes structurelles empêchent certaines personnes de participer à la définition des structures sociales, des institutions auxquelles elles seront incluses et au sein desquelles se poseront leurs actions » (p. 246). Cette conceptualisation associe à la théorie rawlsienne de la justice distributive, une dimension participative et une dimension relationnelle inspirées notamment des travaux de la philosophe féministe Iris Marion Young (2011).

Ce positionnement pour la justice sociale transparaît entre autres dans la question ayant guidé la réalisation de la recherche : "Comment les rapports sociaux, notamment de race, sont-ils organisés et re-produits dans les distributions dissymétriques du travail enseignant? » (Larochelle-Audet, 2019, p. 64). Ainsi formulée, cette question visait à interroger les cadres d'interprétation et les catégories essentialisant les personnes de groupes racisés ${ }^{3}$, dans des configurations propres à la situation sociopolitique québécoise. Elle cherchait également à examiner minutieusement la production et la reproduction des rapports sociaux dans la distribution du travail enseignant, ainsi que l'imbrication complexe du pouvoir dans l'institution éducative. Elle avait, en somme, comme finalité de produire de nouveaux savoirs aptes à provoquer des changements pour plus d'équité dans l'organisation du travail en milieu scolaire. Les plus récents rapports sur l'égalité en emploi de la Commission des droits de la personne et des droits de la jeunesse (CDPDJ) du Québec révèlent en effet que, malgré une progression, le personnel professionnel - une catégorie principalement constituée du personnel enseignant - de « minorités visibles 4 » demeure sous-représenté dans les centres de services scolaires ${ }^{5}$ de la province. En 2019, 6\% du personnel professionnel scolaire du réseau public était de «minorités visibles » (CDPDJ, 2020).

À partir de l'exemple de cette recherche empirique, cet article poursuit deux visées. Premièrement, nous y exposons l'approche épistémo-méthodologique ${ }^{6}$ féministe constituée afin de mener une recherche guidée par les principes des recherches socialement justes. Certains résultats de la recherche sont présentés afin de montrer comment la pensée

\footnotetext{
${ }^{3}$ Rappelons que l'existence de races humaines a été scientifiquement réfutée, mais qu'elles ont historiquement été construites et hiérarchisées pour justifier la domination de certains groupes humains au profit d'autres groupes, notamment au moment de la colonisation et de l'esclavage. Cette construction sociale a encore aujourd'hui des répercussions réelles sur certains groupes sociaux, qui se voient construits et naturalisés sur la base de rapports de domination légitimés par l'idéologie raciste (Guillaumin, 2002). Dans cet article, nous utilisons ainsi le concept de "groupes racisés » pour désigner ces groupes sociaux et de « rapports sociaux de race " pour désigner ce type spécifique de rapports de domination.

${ }^{4}$ Ce terme est utilisé dans les lois pour l'égalité en emploi au Canada. Selon la CPDDJ (2020), " une minorité visible est une personne autre qu'un Autochtone qui n'est pas de race ou de couleur blanche » (p. 64).

5 Appelés auparavant commissions scolaires, les centres de services scolaires sont responsables de l'organisation des services éducatifs à la formation générale et professionnelle, à l'éducation préscolaire et à l'enseignement primaire et secondaire sur un territoire donné.

6 L'expression " approche épistémo-méthodologique » englobe les fondements épistémologiques et les choix méthodologiques privilégiés pour la recherche, tout en montrant l'importance du lien entre les deux dans les recherches qualitatives (Borri-Anadon, 2014).
} 
féministe noire a été mobilisée conjointement à la méthode de l'ethnographie institutionnelle pour faire émerger, depuis la perspective d'enseignantes en insertion professionnelle de groupes racisés, des savoirs qui révèlent et confrontent les pratiques et les idéologies par lesquelles les inégalités sont reproduites au sein de l'institution éducative.

Deuxièmement, nous proposons, en filigrane, une réflexion sur les apports et limites de la démarche de recherche menée pour répondre pleinement aux finalités de justice sociale que nous tentons, humblement, de suivre. Plusieurs questionnements partagés dans le texte laissent entrevoir le travail constant devant être réalisé afin de prendre en compte sa propre position dominante et faire entendre les voix de groupes sociaux généralement tues dans le milieu scientifique. Paradoxalement, nos voix et celles issues de l'académie se retrouvent à plusieurs endroits surexposées dans l'article. C'est donc conscientes de ce fait, que nous tentons de nous positionner dans ce texte en chercheuses solidaires au sens de Piron? (1996). Sans pouvoir les contrôler, nous acceptons la responsabilité de notre texte et de ses conséquences, en tant qu'acte construisant le monde social. Nous acceptons par ailleurs d'apparaître à certains égards moralisatrices, alors que notre texte expose les fondements d'un êthos - manière d'être - (Foucault, 1984a, cité par Piron, 1996) en construction, ayant comme finalité la justice sociale.

\section{DES POSTULATS ÉPISTÉMOLOGIQUES SOCIALEMENT JUSTES}

Dans un ouvrage dédié aux approches de recherche anti-oppression, Potts \& Brown (2015) soutiennent que la réalisation d'une recherche engagée pour la justice sociale implique des postulats épistémo-méthodologiques alternatifs ou engagés au niveau du rapport au savoir, de la posture des chercheurs/euses, de leurs rapports avec les personnes participant à la recherche ainsi que des choix relatifs à la construction et à la conduite de l'enquête (question, méthode, collecte et analyse des données). Que ce soient les perspectives féministes, anti-oppressives, antiracistes, afrocentriques, critiques de la race ("critical race theory »), autochtones, postcoloniales, décoloniales ou du "settler colonialism », plusieurs courants théoriques critiques partagent un engagement commun pour l'émancipation des personnes et le changement social (Strega \& Brown, 2015a). Cette finalité d'émancipation s'inscrit également dans la tradition du paradigme compréhensif où la finalité de l'activité scientifique est orientée par une éthique située et par la contribution au bien commun (Charmillot, 2021).

Le savoir constitue l'enjeu central de la réalisation d'une recherche socialement juste (Strega \& Brown, 2015b), en plus d'être le thème dominant des recherches féministes (Espínola, 2012 ; Olesen, 2011). Les personnes ayant été exclues de la pratique scientifique interrogent la production du savoir : qui est engagé dans la création du sens conféré au monde social ? Comment certaines interprétations plus que d'autres acquièrent-elles le statut de savoirs ?

\footnotetext{
7 Nous saluons la mémoire et la contribution de Florence Piron, partie trop tôt en cette année 2021.
} 
Comment les rapports sociaux de race $^{8}$, de genre et de classe affectent-ils l'organisation du savoir ? Ces questionnements mettent en évidence les inégalités épistémiques caractérisant la recherche en sciences sociales et humaines, se manifestant " dans l'accès, la reconnaissance et la production des savoirs » (Godrie \& Dos Santos, 2017, p. 7). Au-delà des seuls courants féministes, ils s'inscrivent dans une critique multiforme de la prétention à l'universalité, à la neutralité et à l'objectivité dans la recherche en sciences sociales et de ses effets aliénants sur certains groupes sociaux étant traditionnellement tenus aux marges (Collins, 2016).

En ce sens, il est indispensable de « reconnaître de quelle perspective se produit un certain savoir, à partir du centre ou de la marge? » (Benhadjoudja, 2015, p. 51) pour sortir d'une épistémologie de l'ignorance, aveugle aux rapports sociaux et de domination (Strega \& Brown, 2015b). Le savoir émergeant de chaque perspective est différent, et il convient de le rendre explicite. Bien qu'elle soit intimement liée aux positionnements que nous choisissons consciemment d'occuper dans la société pour contribuer à la rendre plus juste, la recherche présentée dans cet article est étroitement liée à nos positions sociales (Anthias, 2008). Elle reflète le point de vue de femmes universitaires du groupe dominant dans les rapports sociaux de race, ce qui se traduit concrètement au Québec par être blanches et francophones à l'accent jugé « conforme » par ce même groupe. Nos positions ont influencé toutes les étapes de la recherche, et notamment les relations que Julie a pu établir avec les personnes lors des entretiens de recherche et les récits auxquels elle a pu avoir accès.

Dans la perspective d'une recherche socialement juste, les chercheurs/euses, en particulier celles et ceux ayant un point de vue externe au groupe à l'étude (" outsider »), ont la responsabilité de prendre les moyens de s'approcher le plus possible des connaissances issues de l'expérience des personnes étant directement concernées par la recherche (Potts \& Brown, 2015). Cet engagement pour le changement social se matérialise notamment par la production de savoirs libérateurs et émancipateurs, c'est-à-dire utiles aux personnes opprimées et à leurs intérêts (Collins, 2016). A minima, les connaissances issues de la recherche ne doivent ni réifier ou renforcer l'oppression et les privilèges, ni reproduire des préjugés et des stéréotypes essentialisant les personnes ou contribuant à en dresser un portrait encore plus stigmatisant (Olesen, 2011 ; Strega et Brown, 2015a).

Suivant ces postulats, un ensemble de questions nous a accompagnées à chaque étape de l'élaboration de notre recherche : est-ce que celle-ci aura comme effet de donner du pouvoir aux enseignantes de groupes racisés ou de leur en retirer ? Est-ce que les savoirs produits seront utiles pour leur émancipation ou contribueront-ils uniquement à l'accroissement des savoirs scientifiques ? Ces questionnements et les postulats épistémologiques présentés nous ont conduites à explorer des approches méthodologiques et des courants théoriques féministes pouvant être mobilisés dans le cadre de notre recherche. La pertinence d'adopter

8 Sur le concept de « race », voir la note en bas de page numéro 3. 
une approche épistémo-méthodologique féministe s'est également avérée au regard de l'importance des rapports sociaux de sexe dans la structuration du travail enseignant en Amérique du Nord et au Québec (Dufour, 1998 ; Dufour \& Dumont, 2004).

\section{UNE MÉTHODE D’ENQUÊTE FÉMINISTE POUR RÉVÉLER LES RAPPORTS DE RÉGULATION}

Notre recherche a pris la forme d'une enquête de type qualitative inspirée par l'ethnographie institutionnelle. Cette méthode sociologique a comme objectif de révéler comment les activités quotidiennes des personnes sont organisées par des rapports de régulation translocaux qui coordonnent et régulent le pouvoir dans les sociétés contemporaines (Smith, 2005 ; 2018). Le projet d'une sociologie se déployant à partir de l'expérience des personnes - et des femmes dans un premier temps - a émergé au moment où l'instigatrice de l'ethnographie institutionnelle, Dorothy E. Smith, a fait l'expérience de la rupture entre la subjectivité de la maison, organisée autour d'une appréhension du monde basée sur le proche et le corporel, et celle de l'université, ayant pour principe de procéder à leur exclusion (Gonzalez \& Malbois, 2013). Pour soutenir l'émergence de savoirs qui n'existent pas « au-delà et au-dessus des gens » (Smith, 2018, p. 50), la sociologue a élaboré une méthode d'enquête féministe qui déplace l'objet d'étude. II ne s'agit pas de mener une recherche sur des personnes occupant une certaine position sociale, ni par ces personnes, mais depuis leur position pour se pencher sur les aspects des institutions ayant à voir avec leurs expériences.

Bien qu'elle soit ancrée dans l'expérience quotidienne des personnes, l'ethnographie institutionnelle ne s'y limite pas. Son point focal est l'étude d'un mode d'organisation sociale de type " textuel » constitué de rapports de régulation ("ruling relations »), un néologisme inventé par Smith (2005) pour désigner des formes objectivées de conscience et d'organisation s'édifiant de manière extralocale, c'est-à-dire externe aux personnes et aux situations concrètes se déroulant ici et maintenant. Comme les rapports de régulation ne peuvent pas être observés en soi, directement, leur découverte se fait par l'étude du travail des personnes et de sa coordination extralocale. Le travail constitue en ce sens une porte d'entrée pour révéler les rapports de régulation limitant la capacité d'agir des individus. Une attention particulière est accordée aux « sources textuelles » - imprimées, mais aussi visuelles, numériques, etc. - coordonnant les activités des personnes, et plus particulièrement au rôle qu'elles jouent « dans la généralisation de l'organisation sociale que nous tenons pour acquise lorsque nous employons le terme d'institution » (Smith, 2018, p. 224). 
Dans le cadre de notre recherche, l'enquête s'est déroulée en trois phases. La première phase visait à recueillir les récits d'informatrices enseignantes ${ }^{9}$ partageant un certain point de vue sur l'insertion professionnelle en enseignement, de par leur position sociale et celle occupée dans l'institution. Notre recherche a ainsi été menée depuis le point de vue de douze femmes et deux hommes de groupes racisés travaillant dans des centres de services scolaires (CSS) francophones de la région métropolitaine de Montréal (RMM), mais n'ayant pas de permanence d'emploi. Sur les quatorze enseignantes, six sont nées au Québec de parents immigrants. Les autres ont immigré il y a plusieurs années, 10 à 12 ans en moyenne, d'Haïti (2), du Maroc (2), de Roumanie (2), de Tunisie (1) et de France (1). Onze enseignantes ont réalisé leur programme de formation initiale à l'enseignement dans une université québécoise (Montréal) et trois ont obtenu une autorisation d'enseigner au Québec à la suite d'une reconnaissance d'équivalences d'une formation réalisée dans une autre province canadienne (1) et dans un autre pays (2). Ces dernières ont aussi réalisé avec succès des exigences de requalification universitaire et une période de probation en milieu scolaire. Au moment de l'entretien, huit enseignantes avaient comme principale occupation l'enseignement dans le réseau public francophone. Trois enseignantes travaillaient à temps partiel en milieu scolaire, tout en réalisant des études de deuxième cycle à temps plein en éducation. Après un passage dans le réseau public, deux enseignantes étaient plutôt à l'emploi d'une école privée et une autre était activement à la recherche d'un emploi après une période de probation.

À partir de ces récits, la deuxième phase consistait à interviewer des personnes ayant un rôle, central ou périphérique, dans l'insertion professionnelle du personnel enseignant, au sein des écoles ou des CSS. Des entretiens ont été menés auprès de 10 informatrices institutionnelles occupant les postes suivants dans deux CSS de la RMM : direction ou direction adjointe d'un établissement d'enseignement (4), employée du secteur des ressources humaines d'un CSS (2), conseillère pédagogique10 d'un CSS (2) et conseillère syndicale (2). Pour approfondir la compréhension des pistes analytiques ayant émergé au cours de ces deux phases, nous avons étudié lors de la troisième phase d'enquête des sources textuelles régulant le travail enseignant au Québec dont des lois, des règlements et des conventions collectives ainsi que d'autres sources produites par des organismes paragouvernementaux.

Au terme des trois phases de l'enquête, l'analyse des matériaux nous a permis de dévoiler un agencement d'activités et de sources textuelles organisant et objectivant l'expérience des enseignantes de groupes racisés sans permanence d'emploi dans le réseau scolaire public (Larochelle-Audet, 2019). Dans l'esprit de l'ethnographie institutionnelle, les résultats de la

\footnotetext{
9 Le terme informatrice est utilisé dans ce texte pour mettre en évidence le rôle des personnes ayant pris part à la recherche. Ces personnes ne sont pas considérées comme des sujets, mais des informatrices pouvant fournir des connaissances pertinentes sur l'expérience se trouvant au cœur de l'enquête ("informants knowledgeable ») (DeVault \& McCoy, 2006). Comme pour le concept d'enseignante, le féminin englobe ici le masculin (voir la note en bas de page numéro 2).

10 Ce travail consiste à conseiller et soutenir les intervenant-es scolaires relativement à la mise en œuvre, au développement et à l'évaluation des programmes d'études, à la gestion de classe et à la didactique.
} 
recherche ont pris la forme d'une cartographie des rapports de régulation, où les personnes vivant une expérience similaire peuvent se situer à la manière d'un point indiquant « vous êtes ici » sur une carte (Smith, 2005). Même s'ils visent en définitive à accroître la capacité des personnes à agir ("empowement»), les savoirs produits au terme de l'enquête tendent à surexposer les activités et textes des groupes dominants dans l'institution éducative et la société, au détriment des expériences partagées par les enseignantes de groupes racisés. Les rapports de régulation ainsi restitués peinent par ailleurs à rendre compte de la complexité et de la fluctuation des rapports de pouvoir émanant des récits des enseignantes, et notamment des multiples configurations du racisme dans l'organisation du travail enseignant.

Ces angles morts de l'ethnographie institutionnelle nous ont amenés à y adjoindre des courants théoriques critiques, comme l'ont fait avant nous plusieurs autres chercheurs/euses (Hampton, 2016). La pensée féministe noire ou féminisme noir ("Black feminism ») a ainsi été choisi11 pour sa capacité à révéler la complexité des systèmes d'oppression, mais également à considérer la parole des personnes étant généralement tenues aux marges de l'académie en tant que savoirs légitimes, essentiels pour organiser la résistance face à l'oppression. Les sections qui suivent rendent visibles les apports spécifiques de ce courant de pensée, d'une part en exposant ses fondements et ancrages et, d'autre part, en partageant des extraits de récits d'enseignantes de groupes racisés ayant pris part à la première phase de notre enquête.

Du point de vue méthodologique, précisons que les récits des informatrices enseignantes ont été recueillis au cours d'entretiens individuels semi-dirigés d'une durée d'environ deux heures lors desquels les informatrices étaient invitées à raconter leur insertion professionnelle en enseignement. Tout en se laissant guider par leur récit, l'intervieweuse - Julie - s'assurait que les principaux thèmes du guide d'entretien soient abordés et que les situations vécues soient décrites de manière suffisamment détaillée. Les entretiens ont été intégralement transcrits, puis analysés selon deux objectifs concomitants typiques de l'ethnographie institutionnelle (McCoy, 2006). Le premier visait à développer une compréhension de l'expérience vécue concrètement par chaque enseignante ("individual's embodied experience »), tandis que le deuxième consistait à rendre visible le champ institutionnel dans lequel ces expériences individuelles sont situées. Ce second objectif s'avère incontournable dans une ethnographie institutionnelle afin d'identifier les sites et les processus à explorer pour la suite de l'enquête et, à terme, faire émerger les rapports de régulation organisant l'expérience étudiée. Cet article se concentre toutefois sur le premier objectif, davantage axé sur la parole et l'expérience des enseignantes.

Pour répondre à ces objectifs, plusieurs étapes analytiques ont été réalisées. Les transcriptions des entretiens ont été d'abord codifiées de manière à y identifier les principales activités institutionnelles caractérisant les expériences vécues, soit l'obtention des

\footnotetext{
11 II s'agit d'un des deux courants mobilisés dans notre recherche, l'autre étant le féminisme matérialiste. Pour en savoir davantage sur cette théorie et la manière dont elle a été utilisée, voir Larochelle-Audet (2019).
} 
autorisations d'enseigner, l'ouverture des dossiers d'employé-es, les entrevues de sélection, l'évaluation des compétences et l'attribution des affectations. Les personnes réalisant ces activités dans l'institution scolaire ainsi que certains éléments biographiques caractérisant les récits des informatrices (ex. parcours migratoire ou positions dans les rapports sociaux) ont également été systématiquement codés. À la suite de ce premier défrichage, une fiche synthèse par informatrice enseignante a été constituée à partir d'extraits décrivant en détail des situations vécues. Les extraits de récits présentés plus loin dans l'article proviennent de ces fiches ${ }^{12}$.

\section{LA PENSÉE FÉMINISTE NOIRE POUR DÉVOILER ET CONFRONTER LES SYSTÈMES D'OPPRESSION}

La pensée féministe noire a émergé aux États-Unis vers les années 1970, face à un mouvement féministe critiqué et reconnu pour son « solipsisme blanc » - d'abord - et à un mouvement antiraciste ignorant le sexisme (Bilge, 2010 ; Dorlin, 2008). Les féministes afroaméricaines montrent alors qu'en s'intéressant seulement au rapport d'oppression genre/ sexe et au système patriarcal, les analyses féministes dominantes tendent à cacher les autres rapports et systèmes de domination (racisme, classisme, hétérosexisme, etc.) (Maillé, 2007). Elles remplacent l'idée de la femme, essentialisée et universelle, par celle de femmes situées au regard d'expériences et de positions occupées, entre autres, dans la division du travail et le système de stratification raciale.

En insistant sur le fait que l'émancipation des femmes ne pourra se faire sans l'émancipation de toutes les femmes, ce courant propose un réel paradigme de l'enchevêtrement des systèmes d'oppression (racisme, sexisme, classisme, etc.) où la domination est appréhendée " comme un système complexe possédant des caractéristiques liées à sa totalité, et des propriétés non déductibles de celles de ses éléments » (Bilge, 2010, p. 59). Les prémisses fondamentales de cette perspective holiste de l'intersectionnalité - une notion de Kimberlé Crenshaw (1989) aujourd'hui bien connue en recherche féministe - prennent ainsi forme au sein du féminisme noir étasunien dans les années 1970 (Bilge, 2009 ; 2010).

Le potentiel émancipateur du féminisme noir se trouve principalement dans son épistémologie féministe afrocentrique, qui « permet de comprendre comment les groupes subordonnés créent un savoir qui résiste à l'oppression » (Benhadjoudja, 2015, p. 51). Le socle de la pensée féministe noire a été construit par des "Africaines-Américaines ordinaires »(Collins, 2008, p. 135), à partir des connaissances issues de leurs expériences concrètes. Son épistémologie prend appui sur la sagesse - en opposition à l'autre type de

\footnotetext{
12 Les autres modes de collecte et d'analyse utilisés - notamment pour le deuxième objectif analytique et les deux autres phases de l'enquête - sont finement décrits dans la recherche doctorale étant à l'origine de cet article (voir Larochelle-Audet, 2019), ainsi que dans un article à paraitre prochainement dans la collection Horssérie «Les Actes » de la revue Recherches Qualitatives suite au Colloque « Usages de perspectives critiques en recherche qualitative : Méthodes, réflexions épistémologiques et questionnements éthiques ».
} 
savoir qu'est l'érudition - qu'elles ont développée afin de vivre et survivre dans un contexte d'oppression raciale, sexuelle et sociale (Collins, 2008). En s'extrayant des cadrages théoriques déjà constitués, cette épistémologie met de l'avant la nature critique des connaissances subordonnées en tant que fondements légitimes afin de lutter pour la dignité humaine et la justice sociale (Collins, 2016).

C'est en ce sens que la section qui suit restitue les récits d'enseignantes de groupes racisés ${ }^{13}$, certaines immigrantes et d'autres nées au Québec, en tant que savoirs essentiels pour résister à l'oppression. II est à noter que nous avons sélectionné uniquement des récits d'enseignantes ayant réalisé un baccalauréat en enseignement au Québec (quatre ans de formation, incluant un an de stage en milieu scolaire) afin de mettre en question le discours selon lequel les difficultés d'insertion professionnelle vécues par le personnel enseignant immigrant sont en large part attribuables aux écarts entre la formation suivie dans leur pays d'origine et celle offerte dans les universités québécoises.

\section{EXPÉRIENCES ET STRATÉGIES D'ENSEIGNANTES POUR RÉSISTER À L'OPPRESSION}

La co-formation ou co-construction des systèmes d'oppression ressort de plusieurs récits d'enseignantes de groupes racisés lorsqu'elles expliquent comment elles se font assigner en milieu scolaire à des catégories figées et contraignantes, contrastant avec les identités qu'elles revendiquent, beaucoup plus complexes et fluctuantes. La perspective ressort notamment des propos de Phany, une femme dans la mi-vingtaine née au Québec, qui s'identifie et se fait assigner à la catégorie « asiatique » :

J'ai vu des parents très surpris de me voir à la rencontre de parents. T'sais, je me suis habituée à ce deux minutes de ... Ils me regardent dans les yeux, puis ils se posent des questions, puis ils s'assoient, puis ils essaient d'agir normalement. Je ne sais pas si c'est juste parce que je suis une femme, asiatique, qui a l'air de 15 ans. Mais c'est peut-être ça. Les trois ! [rires]. (Phany)

Elle explique toutefois que les doutes transparaissant chez les parents d'élèves semblent atténués par les champs dans lesquels elle enseigne:

Moi je suis chanceuse, j'enseigne les maths ! Fait que ça va avec le [rires]... le stéréotype ! Fait que j'ai pas eu tant de réticence à ce niveau-là. On ne m'a pas questionnée par rapport à mes compétences, en mathématiques ni en informatique. (Phany)

En dépit de l'effet de ses champs de spécialisation dans le regard porté sur Phany, des collègues remettent parfois en question l'adéquation entre les différentes dimensions de son identité :

\footnotetext{
13 Issus d'une recherche doctorale, les récits complets peuvent être consultés dans Larochelle-Audet (2019). ॥ est à noter que les prénoms utilisés sont des pseudonymes.
} 
Quand on me demande à mi-conversation : « Est-ce que t'as été adoptée ? " Là je suis : «Pourquoi ? »... «Ben, parce que tu parles bien français. Parce que t'es propre ». «So ? » [...] «Pourquoi est-ce qu'il faut que je sois adoptée? » «Pourquoi ? » [...] Parce que quelqu'un qui vient d'ailleurs ne peut pas être une aussi bonne prof que moi ? C'est ça qui te surprend? Ce qui te surprend c'est que je suis une bonne prof malgré que je suis... asiatique ? Ou ce qui te surprend c'est que... je suis asiatique et que je suis une prof ? Je ne sais pas c'est quoi qui les surprend. Que je sois asiatique ou que je sois bonne prof ? C'est ça qui vient me chercher comme question. Parce que j'ai comme l'impression que comme... c'est pas dans la même phrase : une bonne prof, avec les mentalités véhiculées, et asiatique. (Phany)

Le récit de Pierre propose une autre configuration des systèmes d'oppression dans l'organisation du travail enseignant. Cet homme au début de la trentaine ayant immigré d'Haïti il y a 10 ans et étant assigné Noir en milieu scolaire enseigne l'univers social (géographie et histoire) au secondaire :

Le problème c'est que je suis en histoire du Québec. Quand tu touches I'histoire, c'est comme... Même au niveau de mes amis [...], qui sont Québécois de souche aussi [...] ils comprennent pas que... quelqu'un qui n'est pas Québécois va enseigner l'histoire du Québec. Donc je leur ai dit : "Mais en quoi je ne suis pas Québécois ? C'est quoi ? Je suis tricoté serré, pure laine en plus. Donc... c'est quoi ? » Je sais très bien que... l'évocation du passé est toujours conflictuelle et puis, c'est pas demain que ça va se régler. [...] Les gens trouvent ça louche [rires]. J'ai jamais compris pourquoi [rires]. Parce que moi, j'ai fait mon secondaire à Haïti, puis mon prof c'était un Québécois. [...] J'ai jamais compris pourquoi un enseignant québécois peut m'enseigner l'histoire d'Haïti, moi je peux pas enseigner l'histoire du Québec... [rires]. (Pierre)

Alors que je le questionne sur la façon dont il perçoit son rôle en tant qu'enseignant d'histoire, il explique se sentir davantage surveillé quant aux savoirs qu'il transmet :

Ben moi je suis très bien là-dedans. Je suis capable d'en rire. [...] II fût un temps où j'étais dans une école, puis le directeur-adjoint rentrait chaque cinq minutes pour voir de quoi je parlais ! II y a cette inquiétude-là au niveau du personnel scolaire, pour savoir... Et puis c'est pas juste moi. [...] On disait à la blague [avec des amis] : «Ben c'est tout à fait normal! Regarde-toi ! Qu'est-ce que tu vas dire sur l'histoire du Québec ? » Donc, t'es pas Québécois. [...] Je suis payé pour appliquer le programme, j'applique le programme. Je ne suis pas payé pour enseigner l'histoire des Noirs ou l'histoire d'Haïti, ou whatever! (Pierre)

Sans égard à son champ d'enseignement, Pierre témoigne par ailleurs être régulièrement interpellé comme s'il était le concierge d'une école où il travaille : " J'ai fréquenté une école depuis très longtemps. Puis à chaque fois que je rentre, il y a toujours quelqu'un pour me demander si je suis le nouveau concierge » (Pierre). 
De manière nécessairement différenciée, ces deux récits rendent visibles l'articulation de plusieurs systèmes d'oppression dont le racisme, le sexisme, l'âgisme et le classisme, et de catégories propres à la division du travail en milieu scolaire.

Lors des entretiens, d'autres enseignantes ont partagé différentes stratégies mises en œuvre afin de progresser vers une permanence d'emploi et survivre dans un travail hiérarchisé par rapports sociaux de race. Ces enseignantes relatent notamment être souvent sollicitées pour effectuer le "sale boulot » (Hughes, 1996), comme la suppléance et des remplacements de courte durée. Le récit de Naoual, 30 ans, est particulièrement éloquent à cet égard. Immigrée au Québec au début de la vingtaine d'un pays du Maghreb et s'identifiant et se faisant régulièrement assigner à la catégorie " arabe » dans la société et en milieu scolaire, cette enseignante est diplômée en enseignement au préscolaire-primaire depuis près de deux ans au moment de l'entretien.

N'ayant pas obtenu de contrat au cours de l'année suivant sa diplomation, et ce malgré l'expérience de travail acquise comme suppléante tout au long de ses études en enseignement, Naoual a mis en œuvre deux stratégies en parallèle. D'une part, elle a entrepris d'aider bénévolement une collègue québécoise blanche nouvellement diplômée ayant pour sa part décroché un contrat. D'autre part, elle a directement sollicité les directions d'écoles où des contrats sont encore disponibles, en plus d'utiliser le système centralisé d'affectation du centre de services scolaire (CSS) pour lequel elle travaille :

Ça c'est vraiment la dernière chance quoi. Si tu n'as rien, ben c'est soit tu fais ça, soit... de toute façon tu tentes. [...] Soit tu vas prendre ce qu'on te donne ou tu vas faire de la suppléance jusqu'à ce que quelqu'un t'offre ton contrat. (Naoual)

C'est par ce bénévolat que Naoual parvient finalement à " faire ses preuves » auprès d'une direction d'école, qui lui offre, après une entrevue, son premier contrat (une journée par semaine jusqu'à la fin de l'année) :

La direction, elle a vu le fait que je me suis déplacée, tout ça. Elle m'a dit : « C'est vraiment super qu'il y ait des gens comme toi, bravo pour ton initiative ! » Elle a dit : «Tiens, est-ce que tu es disponible pour faire de la suppléance ? » Je dis : " Oui, absolument ». Elle dit : «Ben je vais te mettre la première sur la liste ». J'ai dit : «C'est gentil ». Et j'ai dit : « Je voulais juste vous dire que hier en fait je vous ai écrit un courriel parce que j'ai vu qu'il y avait des contrats qui restaient ». Elle dit : «Ah oui, malheureusement ces contrats-là ont été comblés. Mais écoute, tu vas être la première que je vais appeler pour la suppléance et dès que j'ai quelque chose... On va essayer de t'intégrer à l'équipe-école ». (Naoual)

Elle postule ensuite à d'autres contrats lui étant devenus accessibles via le système centralisé d'affectation du CSS pour compléter sa tâche, mais sans succès. Pour la première fois, elle se demandera si elle n'est pas discriminée : 
Fait que j'ai été voir et j'avais rien eu. [silence] Et, sérieusement, j'ai... c'est vraiment la première fois que je vais réfléchir de cette façon. Je vais me dire : "De toutes ces personnes-là, ça se peut que ça soit juste une coïncidence, mais... y'a pas une seule personne qui a un nom ... autre qu'un nom québécois ». (Naoual)

Elle obtiendra ensuite d'autres courts remplacements, accompagnés de promesses de meilleurs contrats qui ne se matérialiseront pas :

[L'employée des ressources humaines du CSS] me dit : "Regarde Naoual, si tu prends ça, tu vas être la première que je vais appeler si j'ai quelque chose. [...] » Fait que, elle m'a un petit peu mis dans la situation que je suis comme obligée. [...] Si tu veux avoir de la job ça c'est si jamais je t'appelle - tu vas devoir accepter ça. (Naoual)

Face à ces nombreux culs-de-sac, Naoual a l'impression que ses compétences ne sont reconnues que pour faire des suppléances et des remplacements. Son expérience l'amène à remettre en question l'équité des règles régissant la sélection du personnel enseignant ainsi que l'attribution des affectations :

En façade, c'est tout bien fait, c'est démocratique comme système de sélection. On est hypersélectif, parce qu'on cherche des enseignants compétents. [...] Mais je n'ai pas l'impression qu'il y a des critères clairs et précis dans le recrutement. Y'a des personnes qui n'ont même pas de bacc. Ils ont juste le permis d'enseignement [...] et qui ont des contrats, et qui rentrent sur la liste de priorité. Puis y'a des personnes comme moi, qui ont étudié ici et qui ont quand même de la misère à s'insérer... [rires] vite ! [...] Finalement, on ne sait pas vraiment c'est qui qui choisit, c'est qui qui a le droit, c'est qui qui a la notoriété de décider. (Naoual)

Devant des obstacles similaires, d'autres enseignantes interviewées ont choisi de quitter le réseau scolaire public pour le privé, de changer de champ ou de niveau d'enseignement ou encore d'entreprendre des études supérieures en parallèle. C'est notamment le cas de Pierre, qui nous explique avoir finalement opté pour ce qu'il appelle la stratégie de la girouette :

Pour faire ta place, tu es obligé d'aller là où les autres ont peur d'aller. Et puis, on est conscient du danger. On sait qu'on n'aura pas de cadeau. C'est comme ça. [...] II faut faire les jobs que les gens ne veulent pas faire ! [rires] [...] [Mais,] moi je ne me bats plus avec rien-là. C'est une formule politique [...] : la girouette. Donc... je prends la direction du vent. [rires] Si c'est pas bon maintenant, fine. Je passe à autre chose. On verra dans 10 ans peut-être [rires]. Si j'ai encore du temps. Donc je ne perds pas mon temps à me casser la tête avec des choses inutiles. Je faisais ça quand j'étais plus jeune, mais... là j'ai moins de temps à perdre. (Pierre)

Comme le montrent ces extraits de récits, les stratégies mises en œuvre par Naoual et Pierre pour faire face à l'oppression vécue en milieu scolaire divergent à plusieurs égards. 
Rappelant la perspective holiste de l'intersectionnalité, celles-ci sont intimement liées à leurs positions différenciées dans les systèmes d'oppression et dans la division du travail enseignant. En dépit de leurs différences, elles composent un ensemble de savoirs collectifs essentiels pour organiser la résistance à l'oppression.

\section{CONCLUSION}

Cet article avait comme double visée d'exposer l'approche épistémo-méthodologique féministe constituée dans une recherche empirique pour répondre aux principes des recherches socialement justes, tout en explicitant ses apports et limites à cet égard. Nous avons notamment décrit la méthode de l'ethnographie institutionnelle, et mis en évidence certains de ses angles morts. La pensée féministe noire a été proposée pour répondre à ceux-ci, en fournissant des outils épistémologiques et théoriques rendant visible la coformation des systèmes d'oppression et légitimant l'expérience des enseignantes de groupes racisés en tant que savoirs essentiels pour lutter contre le racisme dans l'organisation du travail enseignant.

Or, le fait que nous mobilisions la pensée féministe noire dans un cadre académique comporte le risque de contribuer à ce que Bilge (2015) appelle « le blanchiment de l'intersectionnalité »:

Par le terme « blanchiment », je fais référence à un ensemble de discours et de pratiques qui évacuent la pensée critique raciale de l'appareillage actuel de l'intersectionnalité et marginalisent les personnes racialisées comme productrices des savoirs intersectionnels des débats et des espaces universitaires contemporains, ainsi qu'à une façon de faire la science qui consolide l'hégémonie au lieu de la déstabiliser. (p. 9)

Ce risque est particulièrement important compte tenu de notre propre position dominante dans les rapports sociaux de race. Bien que nous ayons tenté d'éviter cet écueil en positionnant les voix des enseignantes de groupes racisés à l'avant-plan de cet article, nous ne pouvons pas affirmer l'avoir évité dans notre recherche. Par nos choix épistémologiques, théoriques et méthodologiques, nous avons néanmoins tenté de contribuer à défier et à réduire les inégalités épistémiques plutôt que de les renforcer (Godrie \& Dos Santos, 2017).

Au terme de cette recherche, plusieurs questions relatives à la finalité de transformation sociale étant au cœur de la pensée féministe noire demeurent néanmoins en suspens : estce que les nouveaux savoirs produits contribueront réellement à la réduction des inégalités vécues par les enseignantes de groupes racisés en milieu scolaire et à leur émancipation ? Est-ce que les savoirs constitués à partir des expériences de ces enseignantes trouveront écho dans les milieux scolaires et, le cas échéant, comment seront-ils reçus ? Comment les rapports inégaux de pouvoir et les mécanismes structurels de la reproduction de la domination dans l'institution éducative ayant été documentés pourront-ils être concrètement 
transformés ? Comme nous souhaitons nous positionner en chercheuses solidaires (Piron, 1996) au-delà de ce texte, notre réflexion nous amène actuellement à partir des savoirs des enseignantes recueillis dans cette recherche pour élaborer de nouveaux projets de recherche ayant comme objectif de transformer certaines pratiques ayant cours dans les écoles et les CSS en matière d'insertion professionnelle ou d'embauche. Bien que nous entrevoyons déjà les limites inhérentes à ce type de recherche, nous les considérons comme un moyen d'assumer notre responsabilité en tant que chercheuses de " ce qui est ", mais surtout la possibilité de participer à « ce qui sera » et ainsi insuffler nos préoccupations pour la justice sociale en éducation en milieu scolaire.

\section{RÉFÉRENCES}

Anthias, F. (2008). Thinking through the lens of translocational positionality: An intersectionality frame for understanding identity and belonging. Translocations: Migration and Social Change, 4(1), 5-20.

Benhadjoudja, L. (2015). De la recherche sur les féminismes musulmans : enjeux de racisation et de positionnement. In N. Hamrouni, \& C. Maillé (Eds.), Le sujet du féminisme est-il blanc? Femmes racisées et recherche féministe (pp. 41-56). Montréal, Québec : Remue-ménage.

Bilge, S. (2009). Théorisations féministes de l'intersectionnalité. Diogène, 2009/1(225), 70-88.

Bilge, S. (2010). De l'analogie à l'articulation : théoriser la différenciation sociale et l'inégalité complexe. L'Homme et la société, 2010/2(176-177), 43-64.

Bilge, S. (2015). Le blanchiment de l'intersectionnalité. Recherches féministes, 28(2), 9-32.

Borri-Anadon, C. (2014). Pratiques évaluatives des orthophonistes scolaires à l'égard des élèves issus de minorités culturelles : une recherche interprétative-critique Thèse de doctorat, Université du Québec à Montréal. http://www.archipel.uqam.ca/6351/

Charmillot, M. (2021). Définir une posture de recherche, entre constructivisme et positivisme. In F. Piron (Ed.), Guide décolonisé et pluriversel de formation à la recherche en sciences sociales et humaines (en ligne). Éditions science et bien commun.

https://scienceetbiencommun. pressbooks. pub/projetthese/chapter/les-grands-debatsepistemologiques-occidentaux-attribue/

Collins, P. H. (2008). La construction sociale de la pensée féministe noire. In E. Dorlin (Ed.), Black feminism : anthologie du féminisme africain-américain, 1975-2000 (Traduit par A. Robatel, œuvre originale publiée en 1989, pp. 135-175). Paris : L'Harmattan.

Collins, P. H. (2016). La pensée féministe noire (Traduit et présenté par D. Lamoureux, 2e éd.). Montréal, Québec : Remue-ménage.

Commission des droits de la personne et des droits de la jeunesse (CDPDJ) (2020). Rapport triennal 2016-2019. La loi sur l'accès à l'égalité en emploi dans des organismes publics (publication no 978-2-550-86824-8).

https://www.copdj.qc.ca/Publications/Rapport_triennal_PAE_2016_2019.pdf 
Crenshaw, K. (1989). Demarginalizing the intersection of race and sex: A Black feminist critique of antidiscrimination doctrine, feminist theory, and antiracist politics. University of Chicago Legal Forum, 1989 (Article 8). https://chicagounbound.uchicago.edu/uclf/ vol1989/iss1/8

DeVault, M. L., \& McCoy, L. (2006). Institutional ethnography: Using interviews to investigate ruling relations. In D. E. Smith (Ed.), Institutional ethnography as practice (pp. 15-44). Toronto, Ontario: Rowman \& Littlefield.

Dorlin, E. (2008). Black feminism : anthologie du féminisme africain-américain, 1975-2000. Paris : L'Harmattan.

Duchesne, C. (2017). Quelles stratégies d'acculturation de nouveaux enseignants issus de l'immigration privilégient-ils face aux défis culturels et identitaires de leur insertion professionnelle ? Canadian Journal of Education, 40(1), 1-24.

Dufour, A. (1998). Les institutrices rurales du Bas-Canada : incompétentes et inexpérimentées? Revue d'histoire de l'Amérique française, 51(4), 521-548. https:// doi.org/10.7202/005404ar

Dufour, A., \& Dumont, M. (2004). Brève histoire des institutrices au Québec de la NouvelleFrance à nos jours. Montréal, Québec : Boréal.

Espínola, A. F. (2012). Subjectivité et connaissance : réflexions sur les épistémologies du « point de vue ». Cahiers du Genre, 2(53), 99-120. https://doi.org/10.3917/colge.053.0099

Gagnon, M.-A. (2019, 25 septembre). Toujours aussi peu d'hommes enseignants. Le Journal de Québec.

https://www.journaldequebec.com/2019/09/25/toujours-aussi-peu-dhommesenseignants

Godrie, B., \& Dos Santos, M. (2017). Présentation : inégalités sociales, production des savoirs et de l'ignorance. Sociologie et Sociétés, 49(1), 7-31. https://doi.org/ 10.7202/1042804ar

Gonzalez, P., \& Malbois, F. (2013). La critique saisie par les sociologies pragmatiques. Sur le geste de Dorothy E. Smith (1/2). EspacesTemps.net. https://www.espacestemps.net/ articles/la-critique-saisie-par-les-sociologies-pragmatiques-partie1/

Guillaumin, C. (2002). L'idéologie raciste. Genèse et langage actuel (2e éd.). Paris : Gallimard (CEuvre originale publiée en 1972).

Hampton, R. (2016). Racialized social relations in higher education: Black student and faculty experiences of a Canadian university Thèse de doctorat, McGill University. https:// escholarship.mcgill.ca/concern/theses/q237hv236

Hamrouni, N. (2012). Le care invisible : genre, vulnérabilité et domination Thèse de doctorat, Université de Montréal et Université catholique de Louvain.

https://papyrus. bib.umontreal.ca/xmlui/handle/1866/9059

Hughes, E. C. (1996). Division du travail et rôle social (C. Pierre, trad., œuvre originale publiée en 1956). In E. C. Hughes (Ed.), Le regard sociologique : essais choisis (textes rassemblés et choisis par Jean-Michel Chapoulie) (pp. 62-68). Paris : Éditions de l'École des hautes études en sciences sociales. 
Larochelle-Audet, J. (2017). La transition vers l'emploi des enseignants immigrants : nouvelles perspectives de recherches. Initio, (6), 75-94.

https://www.initio.fse.ulaval.ca/fichiers/site_initio/documents/Volume_1_numero_6/ Larochelle-Audet_INITIO_no._6_printemps_2017.pdf

Larochelle-Audet, J. (2019). Organisation et re-production des rapports de domination dans les distributions dissymétriques du travail enseignant : une enquête du point de vue d'enseignant·es de groupes racisés Thèse de doctorat, Université de Montréal. http:// hdl.handle.net/1866/22439

Maillé, C. (2007). Réception de la théorie postcoloniale dans le féminisme québécois. Recherches Feministes, 20(2), 91-111. https://doi.org/10.7202/017607ar

McCoy, L. (2006). Keeping the Institution in View: Working with Interview Accounts of Everyday Experience. In D. E. Smith (Ed.), Institutional ethnography as practice (pp. 109-126). Lanham, MD: Rowman \& Littlefield.

Morrissette, J., \& Demazière, D. (2018). L'expérience du personnel enseignant formé à l'étranger dans les écoles de Montréal. Apports et enseignements d'une recherche collaborative. Éducation et francophonie, 46(2), 189-207.

https://doi.org/10.7202/1055568ar

Niyubahwe, A. (2015). L'expérience d'insertion professionnelle des enseignantes et enseignants de migration récente au Québec Thèse de doctorat, Université Sherbrooke. https://savoirs.usherbrooke.ca/handle/11143/6972

Olesen, V. (2011). Feminist qualitative research in the millenium's first decade : Developments, challenges, prospects. In N. K. Denzin, \& Y. S. Lincoln (Eds.), The SAGE handbook of qualitative research (4e éd., pp. 129-146). Thousand Oaks, CA: Sage.

Piron, F. (1996). Écriture et responsabilité. Trois figures de l'anthropologue. Anthropologie et sociétés, 20(1), 125-148.

Potts, K. L., \& Brown, L. (2015). Becoming an anti-oppressive researcher. In S. Strega \& L. Brown (Eds.), Research as resistance: Revisiting critical, indigenous and anti-oppressive approaches (2e éd., p. 17-42). Toronto, Ontario: Canadian Scholar's Press.

Rachédi, L., \& Vatz Laaroussi, M. (2021). Revisiter les processus migratoires à la lumière des réalités familiales et des contextes sociopolitiques contemporains. In M. Potvin, M.-O. Magnan, J. Larochelle-Audet, \& J.-L. Ratel (Eds.), La diversité ethnoculturelle, religieuse et linguistique en éducation au Québec. Théorie et pratique (2e éd., pp. 90-104). Montréal, Québec : Fides Education.

Smith, D. E. (2005). Institutional ethnography: a sociology for people. Toronto, Ontario: AltaMira Press.

Smith, D. E. (2018). L'ethnographie institutionnelle : une sociologie pour les gens (Traduit par F. Malbois, M. Barthélémy, \& J. Hedström, œuvre originale publiée en 2005). Paris : Economica.

Strega, S., \& Brown, L. (2015a). Research as resistance: Revisiting critical, indigenous and anti-oppressive approaches (2e éd.). Toronto, Ontario: Canadian Scholar's Press. 
Strega, S., \& Brown, L. (2015b). From resistance to resurgence. In S. Strega, \& L. Brown (Eds.), Research as resistance: Revisiting critical, indigenous and anti-oppressive approaches (2e éd., p. 1-16). Toronto, Ontario: Canadian Scholar's Press.

Young, I. M. (2011). Justice and the politics of difference (Paperback reissue / with a new foreword by Danielle Allen). Princeton, NJ: Princeton University Press.

Open Access Publications - Bibliothèque de I'Université de Genève Creative Commons Licence 4.0

(c) (i) 